\title{
A STUDY OF COST EFFECTIVE SCHEDULING OF NURSES BASED ON THE DOMAIN TRANSFORMATION METHOD
}

\author{
Geetha Baskaran \\ University of Nottingham, Malaysia Campus \\ Jalan Broga , 43500 Semenyih, Selangor \\ E-mail: Geetha.Baskaran@nottingham.edu.my
}

\author{
Andrzej Bargiela \\ University of Nottingham, Nottingham, UK \& \\ Krakow Technical University. Krakow, Poland. \\ E-mail: abb@cs.nott.ac.uk
}

\author{
Rong Qu \\ School of Computer Science \\ University of Nottingham, Jubilee Campus \\ Nottingham NG8 1BB, UK. \\ E-mail: rxq@cs.nott.ac.uk
}

\section{KEYWORDS}

Domain Transformation, Demands, Nurse Scheduling, Granulation, Simulation.

\begin{abstract}
This paper discusses and analyses the tradeoff between the flexibility afforded with greater number of staff and the implied cost of employing extra staff in the context of the nurse-scheduling problem. If the number of staff is constant, our study allows quantification of the degree of pressure put on the staff resulting from the schedules that do not satisfy their preferences for shift allocation. We present a practical approach, based on our domain transformation methodology that achieves good quality schedules without high computational requirements.
\end{abstract}

\section{INTRODUCTION}

A common problem in healthcare systems worldwide is the shortage of nursing staff [Ulrich, 2002]. This is partially due to the demanding nature of the nursing profession that requires availability for shift work. In this context the task of producing work schedules that not only satisfy the clinical cover demands but also take into account specific requests and preferences of nurses, becomes a key to staff satisfaction and retention. A failure to deal with this issue inevitably leads to not having enough skilled nurses in clinical settings and a significant negative impact on patient outcomes, including mortality [Aiken, 2002].

From the perspective of hospital management there is an inseparable link between the scheduling activity and the decision about the total numbers of staff. In their publications [Costa, 1996] and [Knauth, 1996] have provided some guidelines in this respect. However, employing more nurses than is needed to meet the required clinical care standards, is an expense that hospitals are keen to avoid. One way of ensuring that the hospitals strike this delicate balance is to deploy computationally efficient software tools enabling construction of work schedules not only in the long- and medium-term planning mode but also in response to immediate requests from staff.

Independent studies have supported the view that the investment in the advanced scheduling of nursing staff translate into significant enhancement of job satisfaction, and savings in labor costs due to reduced nurse turnover [Bester, 2007, Blythe, 2005]. However, producing schedules that meet hospital requirements and satisfy individual preferences and ad-hoc requests is an extremely complex task [Gino et. Al., 2012].

In this study, we have approached the problem of costeffective scheduling of nurses using the domain transformation method introduced in [Baskaran et al. 2009,2012 ] as a practical illustration of the information granulation methodology [Bargiela et al. 2002, 2008]. Based on this solution we investigate the optimum balance between the staffing levels of the ward and the ability to achieve good quality schedules.

\section{NURSE SCHEDULING PROBLEM}

The nurse-scheduling problem can be defined as a problem of assigning to each nurse a specific shift within a pre-defined scheduling horizon. Such an assignment is subject to hard constraints that originate from contractual agreements, legal requirements and local good practice. Any schedule that satisfies these constraints is referred to as a feasible schedule. However, satisfying hard constraints is only a starting point for the construction of a good quality schedule. The degree of satisfaction of additional constraints reflecting staff preferences for allocation of specific shifts provides a measure of the quality of the schedule.

Without the loss of generality we consider here a nurse scheduling scenario based on the operation of an intensive care units in Dutch hospitals [Baskaran et al. 2009, 2012]. In order to appreciate the computational complexity of the scheduling problem, we consider a specific case of a ward with 16 nurses employed on a 36-hours/week contracts and a scheduling period of 5 weeks (35 days). There are five shift types; as itemized in Table 1, that are allocated to the nurses. The Early (e), Day (d) and Late (1) shifts are of 9-hours duration; 
the Night $(\mathrm{N})$ shift is of 8-hours duration; and the Rest (R) shift lasts a minimum of 2 days.

Table 1: Shift Types and the required numbers of nurses on specific shifts

\begin{tabular}{|c|c|c|c|c|c|c|c|c|c|}
\hline \multirow[t]{2}{*}{$\begin{array}{l}\text { Shift } \\
\text { Type }\end{array}$} & \multirow[t]{2}{*}{$\begin{array}{l}\text { Start } \\
\text { Time }\end{array}$} & \multirow[t]{2}{*}{$\begin{array}{l}\text { End } \\
\text { Time }\end{array}$} & \multicolumn{7}{|c|}{$\begin{array}{c}\text { Number of nurses on } \\
\text { shifts }\end{array}$} \\
\hline & & & $\mathbf{M}$ & $\mathbf{T}$ & $\mathbf{W}$ & $\mathbf{T}$ & $\mathbf{F}$ & $\mathbf{S}$ & $\mathbf{S}$ \\
\hline Early & 07.00 & 16.00 & 3 & 3 & 3 & 3 & 3 & 2 & 2 \\
\hline Day & 08.00 & 17.00 & 3 & 3 & 3 & 3 & 3 & 2 & 2 \\
\hline Late & 14.00 & 23.00 & 3 & 3 & 3 & 3 & 3 & 2 & 2 \\
\hline Night & 23.00 & 07.00 & 1 & 1 & 1 & 1 & 1 & 1 & 1 \\
\hline Rest & \multicolumn{9}{|c|}{ Notional shift that last minimum of 2 days } \\
\hline
\end{tabular}

Night $=\mathrm{N}$ Early $=\mathrm{e} \quad$ Day $=\mathrm{d} \quad$ Late $=1 \quad$ Rest $=\mathrm{R}$

The scheduling problem in the above scenario represents a combinatorial optimisation in a space of $\mathbf{1 6} * \mathbf{5}^{\mathbf{3 5}}=\mathbf{4 . 6} * \mathbf{1 0}^{\mathbf{2 5}}$ possible schedules. Figure 1 illustrates the challenge of the schedulling problem by providing an example of two possible sequences of shifts allocated to Nurse 1 in Week 1. A simple change of one shift implies a non-monotonic change in the cost associated with the violation of constraints.

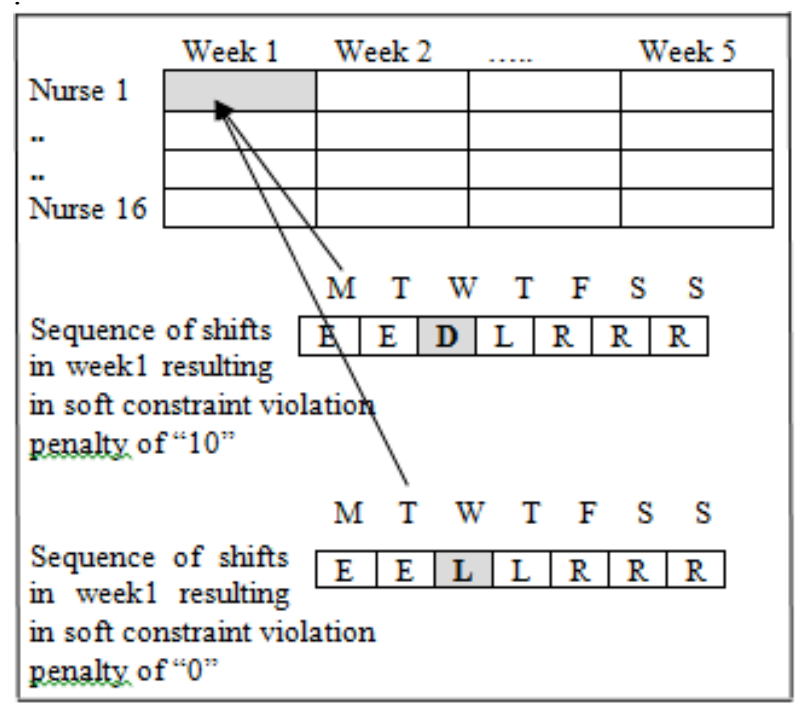

Figure1: Example of a single shift change in a schedule

Because of the large number of possible schedules and the non-monotonic change of the cost with different combinations of shifts, the optimisation of the overall schedule by the modification of individual shifts and/or various groups of shifts is considered as an NP-hard problem [Celia et. al., 2010]. However, this classification is predicated on the assumption of the deployment of scheduling algorithms that explore directly the solution space. An overview of such algorithms is given in [Burke et al., 2004, Lim et al., 2010].

\section{METHODOLOGY}

The domain transformation approach introduced in [Baskaran et al. 2012] departs from the orthodoxy of direct exploration of the space of schedules, as described in the preceeding section. We observe that the three 9-hour shifts (e, d, l) are subject to identical soft constraints. Consequently, the first simplification of the scheduling problem is afforded by considering the e-, $\mathrm{d}-$ and 1-shifts as being of the same type. We denote this combined shift as D-shift and will refer to this transformation as transformation from the edlNR domain to the DNR domain.

In the DNR domain the requirement for staff cover during the corresponding shifts is summarised in Table 2. This in itself does not have any adverse effect on the computational complexity of the scheduling process. However, the important gain is that the reduction of the number of shifts from 5 to 3 makes the number of possible schedules in the DNR domain reduce to $16 * 3^{35}=8 * 10^{17}$. This represents a reduction by a factor of $10^{8}$. But although the application of the traditional scheduling methods would be much more efficient in this reduced space we do not advocate the use of these methods as we notice a potential for additional domain transformation and the associated computational gain.

Table 2: Shift Types and the required numbers of nurses on specific shifts in the DNR domain

\begin{tabular}{|l|l|l|l|l|l|l|l|}
\hline $\begin{array}{c}\text { Shift } \\
\text { type }\end{array}$ & \multicolumn{6}{|c|}{ Number of nurses on specific shifts } \\
\hline & M & T & W & T & F & S & S \\
\hline d & 9 & 9 & 9 & 9 & 9 & 6 & 6 \\
\hline N & 1 & 1 & 1 & 1 & 1 & 1 & 1 \\
\hline R & \multicolumn{6}{|l|}{ Notional shift that last minimum of 2 days } \\
\hline
\end{tabular}

We note that the soft constraints are expressed in therms of penalties associated with specific shift sequences during one week. We can therefore produce sequences of shifts of one-week duration that do not have any penalties associated with them and sequencies that have some arbitrary penalties. We will call those sequencies "patterns" and we will use them as basic building blocks for the schedules. Figure 2 provides examples of such zero-cost patterns and Figure 3 provides examples of non-zero-cost patterns.

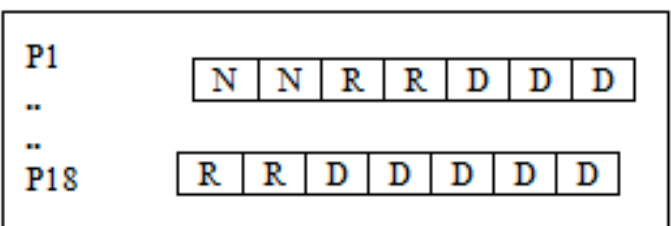

Figure2: No violation of Soft constraints (called as zero cost patterns) 


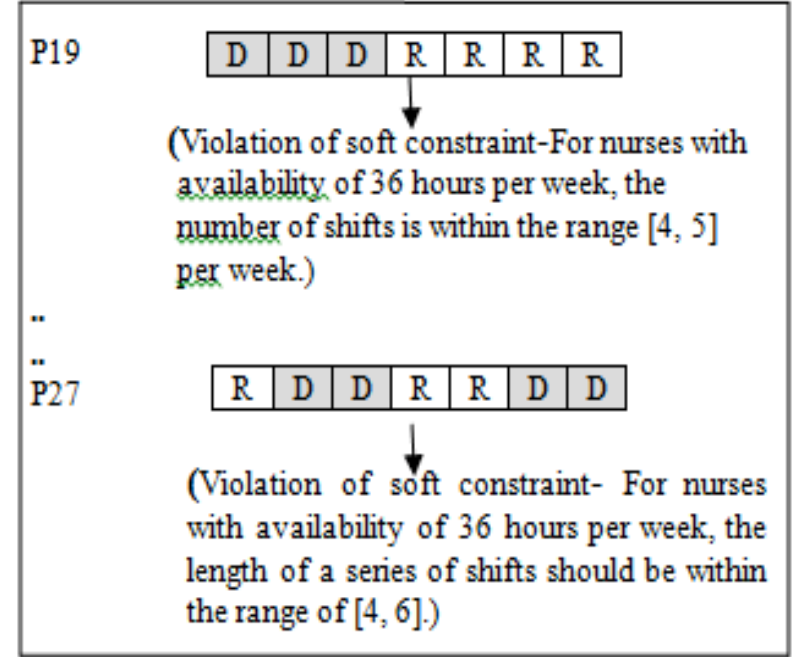

Figure3: Violation of Soft constraints with Cost 10 (called as non-zero cost patterns)

The problem of scheduling shifts is therefore transformed into the problem of scheduling patterns. The computational gain that can be attained from this domain transformation depends on the number of patterns that need to be considered. It turns out that the number of zero-cost patterns and patterns with other pre-specified cost is relatively small. In the scenario considered above there are only 18 zero-cost patterns. This means that there are only $16 * 18^{5}=3 * 10^{7} 5$-week schedules that can be constructed from 18 patterns for 16 nurses. This is a number of schedules that can be completely enumerated within seconds on an average PC.

It is worth highlighting that the combined two domain transformations have achieved an enormous reduction of the space of possible schedules by a factor $10^{18}$. In other words, one second of computations in the domain of patterns is equivalent to $100,000,000,000$ years of computations in the edlNR domain. Of course, the solution of the scheduling problem in the domain of patterns needs to be converted back into the original edINR domain. This involves small computational effort primarily concerned with the consideration of the specific requirements with regard to the precedence of e-, d- and 1-shifts as summarised in Table 2.

Table 2: Penalties of violation for the conversion of DNR domain to edINR domain.

\begin{tabular}{|l|l|l|l|l|l|}
\cline { 3 - 6 } \multicolumn{2}{c|}{} & \multicolumn{4}{c|}{ Succeeding shifts } \\
\cline { 3 - 6 } \multicolumn{2}{c|}{} & N & E & D & L \\
\hline \multirow{3}{*}{$\begin{array}{l}\text { Preceding } \\
\text { Shift }\end{array}$} & N & ok & n/f & n/f & n/f \\
\cline { 2 - 6 } & E & ok & ok & ok & ok \\
\cline { 2 - 6 } & D & ok & 5 & ok & ok \\
\cline { 2 - 6 } & L & ok & n/f & n/f & ok \\
\hline
\end{tabular}

Our domain transformation approach can be summarised as a 3-stage process:

I) conversion of the problem from the original
edINR domain into a problem in the $D$ NR domain; II) solution of the problem in the $D \mathrm{NR}$ domain

III) conversion of the $D \mathrm{NR}$ solution into a solution in the original edINR domain

In practice we are able to achieve further gains in performance by considering the patterns with $\mathrm{N}$-shifts first and, by doing so, reducing the number of nurses that need to be allocated patterns with D-shifts.

\section{BALANCING THE COST OF SOFT CONSTRAINTS AND THE STAFF COST}

Nurse scheduling is inextricably linked with determining total number of nurses. Most of healthcare systems are under pressure to control costs while trying to provide high levels of service. This is a difficult balance to strike. Having a small number of nurses may impact quality of care while employing a large number of nurses and not utilizing their contractual hours is clearly wasteful. In our approach, we are balancing these concerns by combining the cost for the underutilization of nurses with the costs of violation of soft constraints into a single performance index.

The expectation is that with the decreasing number of nurses we will find a progressively higher cost of violation of constraints up to the point when hard constraints would have to be broken - thus no feasible solution could be found. On the other hand, with the increasing number of nurses we expect that although it becomes easier to find schedules that don't violate soft constraints (i.e. one may find low- or zero- cost schedules) the pro-rata cost of unused contractual hours of extra nurses dominates the balance.

For the sake of clarity we demonstrate our balancing approach only in the context of full time nurses employed on 36 hours/week contracts. The underutilisation of nurses $(\mathrm{U})$ is measured as:

where:

$$
\mathrm{U}=\mathrm{TC}-\mathrm{TW}
$$

TC is the Total Number of contractual hours per week TW is the Total Number of hours worked by all nurses in one week (as defined by the shift-cover requirement)

\section{Process of checking the demands}

It is important to appreciate that the number of hours defined by the shift-cover requirement (TW) does not determine, on its own, the required staff numbers. A simple division of TW by the number of hours per week stipulated by the employment contract provides only a lower bound on the number of the required staff but it does not provide a good estimate of the actual staffing 
requirement. This is because the varying (hard and soft) constraints, may imply the need for extra staff despite identical shift-covers and nurse contracts.

For the purpose of balancing the nurse schedules and staff numbers we consider only positive values of $U$ in equation (1). This is because the negative numbers represent the requirement that a nurse works longer hours then implied through the contract and this is already penalizes through the hard/soft constraints. The comparison of the cost associated with violation of constraints with the monetary cost of employing extra staff requires adoption of some convention that would make these costs comparable. Here we assume that the following represent $\mathrm{s}$ well the notional cost of underemployment of staff:

$$
\mathrm{CU}=\mathrm{U} * 10
$$

where:

$\mathrm{CU}=$ Cost of under- utilization

\section{NUMERICAL RESULTS}

Numerical experiments described in this section provide a representative sample of the simulation studies conducted to balance the degree of satisfaction of soft constraints vs. the decisions on employing additional nursing staff. We have varied the required cover on individual shifts to simulate the decision support functionality that may be required by the hospital management. As discussed in the previous section, the total number of schedules in the re-defined problem domain of patterns is of the order of $3 * 10^{7}$ and the solution can therefore be found by enumeration of schedules and the selection of the lowest cost solution.

In order to understand the behaviour of our model, multiple demand vs. number of nurses scenarios were generated. For each scenario, the solution time is also calculated in seconds using a Workstation with Intel ${ }^{\circledR}$ Core TM Dual Quad CPU Q8200@2.33Ghz 4Core running Windows 7 Ultimate with 4GB.

\section{Test Data on Original Demand}

Based on the original problem, we have changed few sample runs of different number of nurses Table 3 presents the results of the best set of nurses which satisfies the demand of the original problem with a very reasonable cost for a month. While Table 4 and 5 represents the alternative demands with the number of nurses and however this is concluded in the Graph 1, Graph 2 and Graph 3 which shows clearly the representation of the various cost.

The results tables use the following notation:

$\mathrm{TN}=$ Total number of nurses

$\mathrm{TC}=$ Total number of contractual hours

$\mathrm{U}(\mathrm{h} / \mathrm{w})=$ Under Utilization of Nurses (hours/week)

$\mathrm{CSC}=$ Cost of violating Soft constraint

$\mathrm{CU}=$ Cost of under- utilization
$\mathrm{T}(\mathrm{s})=$ Time (in seconds) to execute the software

\section{Case 1:}

Table 3: The balance of violation of soft constraints and the underutilisation of nurses for the " 9999966 " D-shift and the " $1111111 "$ N-shift cover $(57 * 9+7 * 8=569$ hours)

\begin{tabular}{|c|l|l|l|l|l|l|}
\hline TN & TC & $\begin{array}{l}\text { U } \\
(\mathbf{h} / \mathbf{w})\end{array}$ & CSC & CU & Ctot & T(s) \\
\hline 14 & 504 & 0 & 1250 & 0 & 1250 & 706 \\
\hline 15 & 540 & 0 & 375 & 0 & 375 & 503 \\
\hline 16 & 576 & 7 & $\mathbf{2 1 0}$ & $\mathbf{7 0}$ & $\mathbf{2 8 0}$ & 139 \\
\hline 17 & 612 & 43 & 185 & 430 & 615 & 31 \\
\hline 18 & 648 & 79 & 180 & 790 & 970 & 22 \\
\hline
\end{tabular}

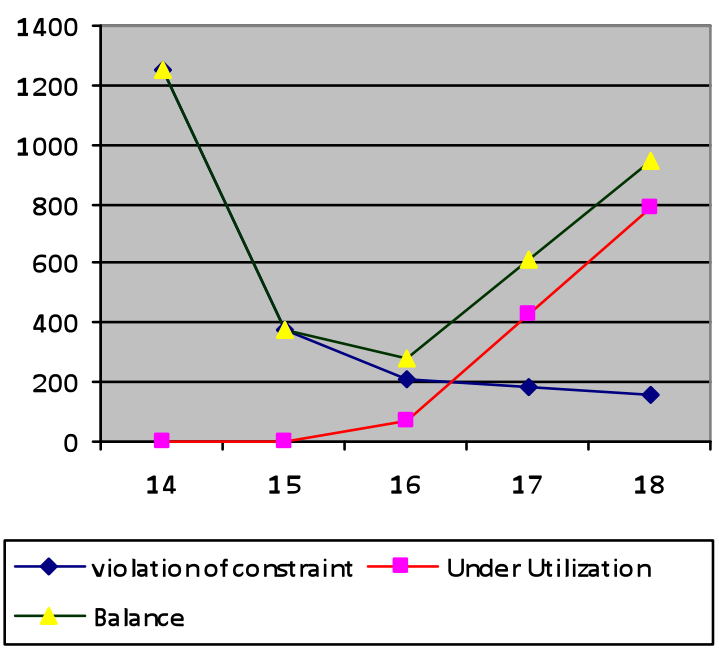

Graph 1: Balance between the constraint cost and the Under Utilization cost for the cover "9999966"

\section{Case 2:}

Table 4: The balance of violation of soft constraints and the underutilisation of nurses for the " 8888855 " D-shift and the " $1111111 " \mathrm{~N}$-shift cover $(50 * 9+7 * 8=506$ hours)

\begin{tabular}{|l|l|l|l|l|l|l|}
\hline TN & TC & $\begin{array}{l}\text { U } \\
\text { (h/w) }\end{array}$ & CSC & CU & Ctot & T(s) \\
\hline 14 & 504 & 0 & 2854 & 0 & 2854 & 761 \\
\hline 15 & 540 & 34 & $\mathbf{3 4 5}$ & $\mathbf{3 4 0}$ & $\mathbf{6 8 5}$ & 501 \\
\hline 16 & 576 & 70 & 290 & 700 & 990 & 204 \\
\hline 17 & 612 & 106 & 190 & 1060 & 1250 & 71 \\
\hline 18 & 648 & 142 & 160 & 1420 & 1580 & 73 \\
\hline
\end{tabular}




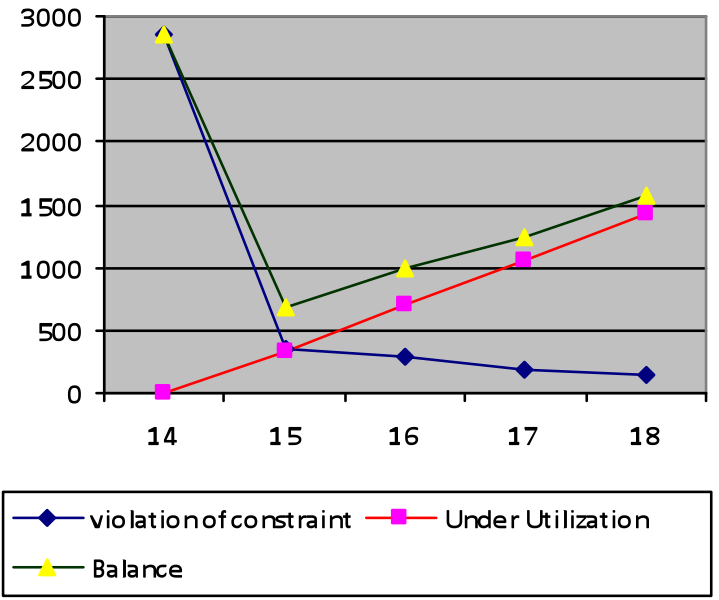

Graph 2: Balance between the constraint cost and the Under Utilization cost for the cover " 8888855 "

\section{Case 3:}

Table 5: The balance of violation of soft constraints and the underutilisation of nurses for the "101010101077" D-shift and the "1111111" N-shift cover $(64 * 9+7 * 8=632$ hours $)$

\begin{tabular}{|c|l|l|l|l|l|l|}
\hline TN & TC & $\begin{array}{l}\text { U } \\
(\mathbf{h} / \mathbf{w})\end{array}$ & CSC & CU & Ctot & T(s) \\
\hline 14 & 504 & 0 & 7175 & 0 & 7175 & 863 \\
\hline 15 & 540 & 0 & 4350 & 0 & 4350 & 809 \\
\hline 16 & 576 & 0 & 3900 & 0 & 3900 & 790 \\
\hline 17 & 612 & 0 & 2550 & 0 & 2550 & 779 \\
\hline 18 & 648 & 16 & $\mathbf{3 0 0}$ & $\mathbf{1 6 0}$ & $\mathbf{4 6 0}$ & 504 \\
\hline 19 & 684 & 52 & 355 & 520 & 875 & 515 \\
\hline 20 & 720 & 88 & 430 & 880 & 1310 & 641 \\
\hline
\end{tabular}

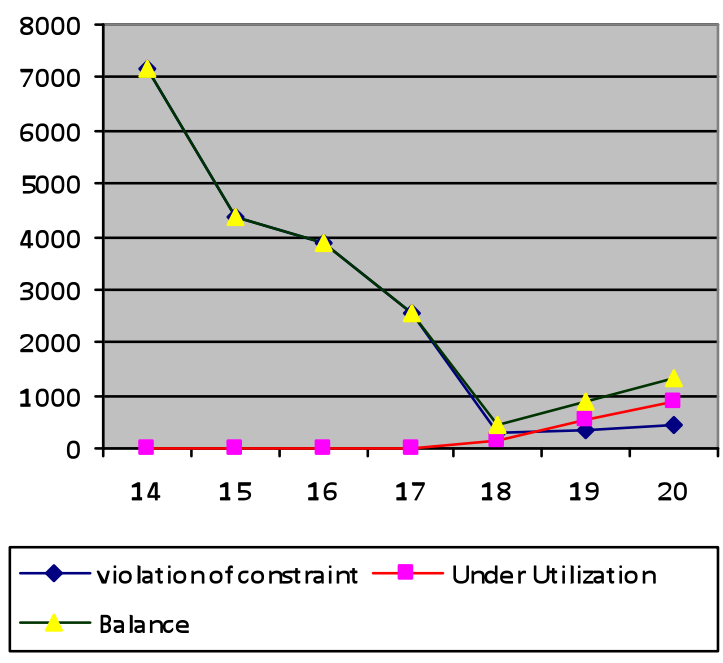

Graph 3: Balance between the constraint cost and the Under Utilization cost for the cover "101010101077"

\section{Discussion}

This study illustrates our investigation of the combined nurse schedulling and the management decision support concerning the staffing levels. The study quantifies how the constraints associated with the scheduling problem influence the cost-effectiveness of employing additional staff. We have shown this using a representative set of 3 different scenarios with different number of nursing staff considered in each scenario.

The result indicates that for the original problem demand of "999966" D-shift, the exact balance is 16 nurses, as indicated in the graph 1 . With fewer then 16 nurses we can't satisfy the clinical cover requirement and having larger numbers of nurses implies unnecessarily high employment cost.

The balance for the alternative clinical cover requirements (demands) of " 8888855 " is 15 nurses; and for the "101010101077" the required numbrt of nurses is 18 .

\section{CONCLUSION}

Nurse rostering problem, considered at the level of detailed time constraints and different types of dayshifts, represents a very significant computational challenge. In this paper we evaluate the effectiveness of our domain transformation approach to achieving a good balance between staffing and the satisfactory work schedules. By automating the nurse rostering process we have reduced the scheduling effort and have ensured that specific scheduling requirements of staff are taken into consideration more easily.

The proposed domain-transformation approach to nurse rostering represents a significant departure from the heuristic/metaheuristic approaches that rely on the randomisation of the search procedure in the vast search space. By contrast, out approach offers deterministic reproducibility of solutions since the domain of patterns allows for full enumeration of solutions. However, it must be noted, that although our proposed method provides competitive and transparent results, it does not guarantee the global optimum. This is because the selection of non-zero cost patterns that are used in a specific scheduling process is guided only by a (rational) notion of making use of cheaper patterns first.

\section{REFERENCES}

Aiken, L.H, Clarke, S.P, Sloane, D.M, Sochalski, J and Silber, J.H. 2002. "Hospital nurse staffing and patient mortality, nurse burnout, and job dissatisfaction," The Journal of American Medical Association. vol. 288. no. 16. pp. 1987-1993.

Bard J. and Purnomo H.W. 2005. "Preference Scheduling for Nurses using Column Generation”. EJOR. 164: 510534.

Bargiela A., 1985. "An algorithm for observability determination in water-system state estimation", IEEE Proceedings Part D. 132. 245-249. 
Bargiela, A., and Pedrycz, W. 2002. "Granular Computing An Introduction”, Kluwer Academic Publishers. 2002. http://dx.doi.org/10.1007/978-1-4615-1033-8

Bargiela, A. and Pedrycz, W. 2008. "Toward a theory of Granular Computing for human-centred information processing", IEEE Trans. On Fuzzy Systems. 16(2): 320-330. http: //dx.doi.org/10.1109/TFUZZ.2007.905 912

Baskaran Geetha, Bargiela Andrzej, Qu Rong. 2009. "Hierarchical method for nurse rostering based on granular pre-processing of constraints", Proc. 23rd European Conference on Modelling and Simulation, ECMS, Madrid, Spain, pp.855-861, June 2009, (doi: 10.7148/2009-0855-0861).

Baskaran, G., Bargiela, A., Qu, R. 2012. "From Simplified to Detailed Solutions to the Nurse Scheduling Problem", 25th European Conference on Operational Research, EURO, Session TC-14, p.134-135, Vilnius, Latvia, July 2012.

Bester, M, Nieuwoudt, I. and Vuuren, J.H.V. 2007. "Finding good nurse duty schedules: a case study", Journal of Scheduling. vol. 10. no. 6. pp. 387-405,

Blythe, J, Baumann, A, Zeytinoglu, I, Denton, M. and Higgins, A. 2005. "Full-Time or Part-Time Work in Nursing: Preferences, Tradeoffs and Choices", Healthcare Quarterly. vol. 8. no. 3. pp. 69-77.

Burke E., De Causmaecker P., Vanden Berghe G. and Van Landeghem H.2004. "The State of the Art of Nurse Rostering". Journal of Scheduling. 7(6). 441-499.

Chase, M. and Kohlweiss, M. 2011. "A Domain Transformation for Structure-Preserving Signatures on Group Elements", IACR Cryptology ePrint Archive 2011: 342 .

Celia, A.G. and Roger, A.K. 2010. " The nurse rostering problem: A critical appraisal of the problem structure", Europeran Journal of Operational research 202(2010):379-389. (doi:10.1016/j.ejor.2009.05.046)

Cheung, G., Ishida, J., Kim, W., Kubota, A., Ortega, A. 2011. "Transform Domain Sparsification of Depth Maps using Iterative Quadratic Programming," IEEE International Conference on Image Processing, Brussels, Belgium, September 2011.

Cheung, G., Kim, W., Ortega, A., Ishida, J., Kubota, A. 2011."Depth Map Coding using Graph Based Transform and Transform Domain Sparsification", IEEE International Workshop on Multimedia Signal Processing, Hangzhou, China, October 2011

Costa, G. 1996. " The Impact of Shift and Night Work on Health", Appl. Ergonomics. 27. 9-16.

Gino J.L. , Mobasher, A., Murray J.C., 2012. "Multi-objective Nurse Scheduling Models with Patient Workload and Nurse", Preferences Management 2012. 2(5): 149-160 DOI: $10.5923 / \mathrm{j} . \mathrm{mm} .20120205 .03$.

Isken, M.W. and Hancock, W.M. 1998. "Tactical staff scheduling analysis for hospital ancillary units". J Soc Health Syst 5:11.

Knauth, P. 1996. “ Design Better Shift System”, Appl. Ergonomics. 27. 39-44.

Kim, J., Jones, K. and Horowitz, M. 2007. "Variable domain transformation for linear PAC analysis of mixedsignal systems", Paper presented at the International Conference on Computer-Aided Design. ICCAD , 05 - 08 Nov 2007. San Jose, California, USA.

Lim, H.T., and Ramli, R. 2010. "Recent Advancements of Nurse Scheduling Models and A Potential Path", Proceedings of the 6th IMT-GT Conference on Mathematics, Statistics and its Applications
(ICMSA2010). Universiti Tunku Abdul Rahman, Kuala Lumpur, Malaysia

Payne, J.F, Bruce, B.B, Lee, L.B, and Yeh, S. " Logarithmic transformation of spectral-domain optical coherence tomography data in uveitis-associated macular edema. Investigative Ophthalmology \& Visual Science, November 2011, Vol. 52, No. 12: 89398943.

Pedrycz W, Smith M.H., Bargiela A. 2000. "Granular signature of data", Proc. 19th Int. (IEEE) Conf.NAFIPS'2000, Atlanta. July 2000; 69-73. http://dx.doi.org/10.1109/NAFIPS.2000.877387

Stanley, L. 2001. "Optimal Shape Design Using Domain Transformations and Continuous Sensitivity Equation Methods", System Modelling and Optimization. Volume 258 of IFIP Conference Proceedings. page 301-316. Kluwer.

Ulrich, C. et al. 2002. "The nursing shortage and the quality of care,", The New England Journal of Medicine. vol. 347. no. 14. pp. 1118-1119.

\section{AUTHOR BIOGRAPHIES}

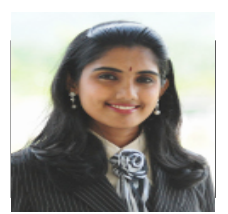

GEETHA BASKARAN was born in

Melaka, Malaysia. She is an Assistant Professor at the University of Nottingham, Malaysia Campus. She is a member of the Automated Scheduling and Planning research group in the School of Computer Science at the University of Nottingham. She is currently pursuing her $\mathrm{PhD}$ studies here focussing on Nurse Scheduling. Her main research area include nurse scheduling, domain transformation, information granulation, heuristic, IP.ILP, matrix exploration.

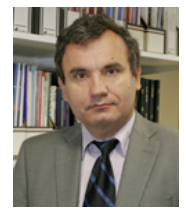

ANDRZEJ BARGIELA is Professor in the School of Computer Science at the University of Nottingham. He served as President of the European Council for Modelling and Simulation (ECMS) during 2002-2006 and 2010-2012.

He is Associate Editor of the IEEE Transactions on Systems Man and Cybernetics and Associate Editor of the Information Sciences. His research involves investigation into Granular Computing, human-centred information processing as a methodological approach to solving large-scale data mining and system complexity problems.

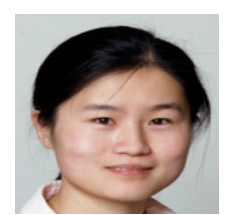

DR RONG QU is a Lecturer in the School of Computer Science at the University of Nottingham. She gained her $\mathrm{PhD}$ in Computer Science from the University of Nottingham in 2002. Her main research areas include meta-heuristics, constraint programming, IP/ILP, case based reasoning methodologies and knowledge discovery techniques on scheduling, especially educational timetabling, healthcare personnel scheduling, network routing problems and graph colouring. In total she has more than 30 papers published or to appear at international journals and peer-reviewed international conferences. $\mathrm{Dr} \mathrm{Qu}$ is also a guest editor for special issues at the journal of Memetic Computing and the Journal of Scheduling, and the program chair of several workshops and an IEEE symposium. 